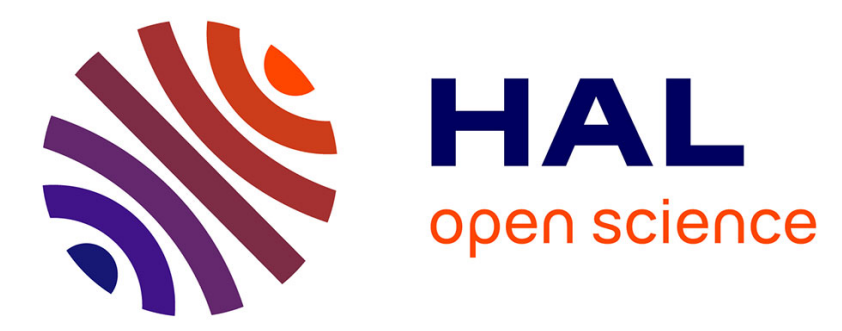

\title{
Reconfigurable Microwave Components Using Glide-symmetric Pin-loaded Parallel Plates
}

Mohammad Bagheriasl, Julien Sarrazin, Guido Valerio

\section{To cite this version:}

Mohammad Bagheriasl, Julien Sarrazin, Guido Valerio. Reconfigurable Microwave Components Using Glide-symmetric Pin-loaded Parallel Plates. Conference EuCAP 2020, Mar 2020, Copenhagen, Denmark. pp.1-4, 10.23919/EuCAP48036.2020.9135362 . hal-03152000

\section{HAL Id: hal-03152000 \\ https://hal.sorbonne-universite.fr/hal-03152000}

Submitted on 25 Feb 2021

HAL is a multi-disciplinary open access archive for the deposit and dissemination of scientific research documents, whether they are published or not. The documents may come from teaching and research institutions in France or abroad, or from public or private research centers.
L'archive ouverte pluridisciplinaire HAL, est destinée au dépôt et à la diffusion de documents scientifiques de niveau recherche, publiés ou non, émanant des établissements d'enseignement et de recherche français ou étrangers, des laboratoires publics ou privés. 


\title{
Reconfigurable Microwave Components Using Glide-symmetric Pin-loaded Parallel Plates
}

\author{
Mohammad Bagheriasl*, Julien Sarrazin*, Guido Valerio*, \\ *Laboratoire d'Électronique et Électromagnétisme, Sorbonne Université, Paris, France, mohammad.bagheriasl@ sorbonne-universite.fr
}

\begin{abstract}
Glide-symmetric structures have recently gained a lot of interest in the design of electromagnetic bandgap materials due to their high attenuation in the stopband region and for their capability to support an almost dispersionless wave propagation. In this paper, we propose a reconfigurable waveguide using a glide-symmetric structure with pins. We show how the wave propagation in this waveguide can be enabled or suppressed by a mere adjustment of the displacement between the two metallic plates of the waveguide. In addition, we demonstrate how this structure can be used to design a phase shifter.
\end{abstract}

Index Terms-electromagnetics, propagation, waveguide, phase shifter, periodic structure, glide symmetry.

\section{INTRODUCTION}

Among the several metasurface configurations proposed in the last years, one of the most common ones is the bed of nails [1], [2]. It has been widely used in gap waveguide technology in order to form an electromagnetic bandgap (EBG) material mimicking a perfect magnetic conducting wall (PMC) [3], [4], [5], [6]. Recently, glide-symmetric bed of nails configurations have gained interest due to the specific characteristics of periodic structures with glide symmetry [7], [8], [9]. The structures exhibiting glide symmetry are invariant under the composition of a translation and a mirror reflection. These structures have wider and stronger stopbands compared to nonglide-symmetric periodic structures. Therefore, different glidesymmetric configurations have recently been used in gapwaveguide technology to further enhance the EBG stopband properties [10], [11]. Furthermore, glide-symmetric structures show lower dispersion compared to non-glide-symmetric structures. This makes such structures a good candidate for wave-guiding applications too. A recent design has shown a phase shifter in gap waveguide technology with a glidesymmetric holey EBG using different number of dielectric layers at a time [12]. In this paper, first we propose to use a waveguide filled with a glide symmetric pin-like medium and confined with an EBG glide-symmetric pin-like region. Then, we show how we can take advantage of the contactless plates to switch the waveguide propagation features by mechanically adjusting the distance between them. This can be used to reconfigure the propagation path of a signal for the design of a switch which feeds different input ports of a multibeam antenna [9]. Finally, we show how it is possible to design a phase shifter without the use of a dielectric layer to create the required phase shift. All the results are validated with simulation performed with CST Microwave Studio.
In Sec. II, we introduce the periodic structure used for our reconfigurable components. In Sec. II-A, we show the dispersion diagrams for the waveguide and the EBG regions to display their passband and stopband characteristics at the desired frequencies respectively. Then, we discuss how the gap between the pins and the metallic plate can be used to control the stopband characteristic of the structure in order to design a reconfigurable waveguide. In Sec. II-B, we explain how this design can be used to form a phase shifter.

\section{Reconfigurable Microwave component Design}

We use here a glide-symmetric pin-loaded parallel-plate waveguide (PPW) to design our reconfigurable guiding structure. The unit cell of the periodic structure is shown in Fig. 1 (a). Since there is no contact between the top and the bottom plate, we have a freedom of choice for the gap $g$ between the pin and the other plate. In Sec. II-A, we exploit the contactless technology and show how the gap $g$ between the pin and the opposite plate can be adjusted to achieve reconfigurability of the wave propagation. The glide symmetry is chosen to obtain low dispersion for the guided wave and at the same time a strong attenuation in the EBG part of our structure. We therefore use the same kind of unit cell shown in Fig. 1 (a) to form the waveguide and the EBG, and we choose different geometrical parameters for the two regions. To design the inner region of the waveguide, we will use a dense arrangement of pins (small period $p$ ), which corresponds to a pass-band medium. We surround this region between regions with lower density of pins (larger period $p$ ) to suppress the cross-sectional wave propagation, which corresponds to an EBG medium. Fig. 1 (b) shows the top view of the structure that we study in this paper, where two straight waveguides are integrated in the same PPW. One can observe that we have used two denser regions (waveguides) in between three less dense regions (EBG) to form two adjacent waveguides.

\section{A. Waveguide}

The parameters for the dense regions (the guiding regions) are $p=1.5 \mathrm{~mm}, h=1.2 \mathrm{~mm}, d=0.5 \mathrm{~mm}$ and $g=0.25 \mathrm{~mm}$. Fig. 2 plots the dispersion diagram of this region in blue color. The parameters for the EBG regions are $p=2.2 \mathrm{~mm}, h=$ $1.2 \mathrm{~mm}, d=0.5 \mathrm{~mm}$ and $g=0.25 \mathrm{~mm}$. Fig. 2 displays the dispersion diagram of the EBG unit cell in red color. We see that by increasing the period of the unit cell from $p=$ $1.5 \mathrm{~mm}$ to $p=2.2 \mathrm{~mm}$, we have increased the stopband 

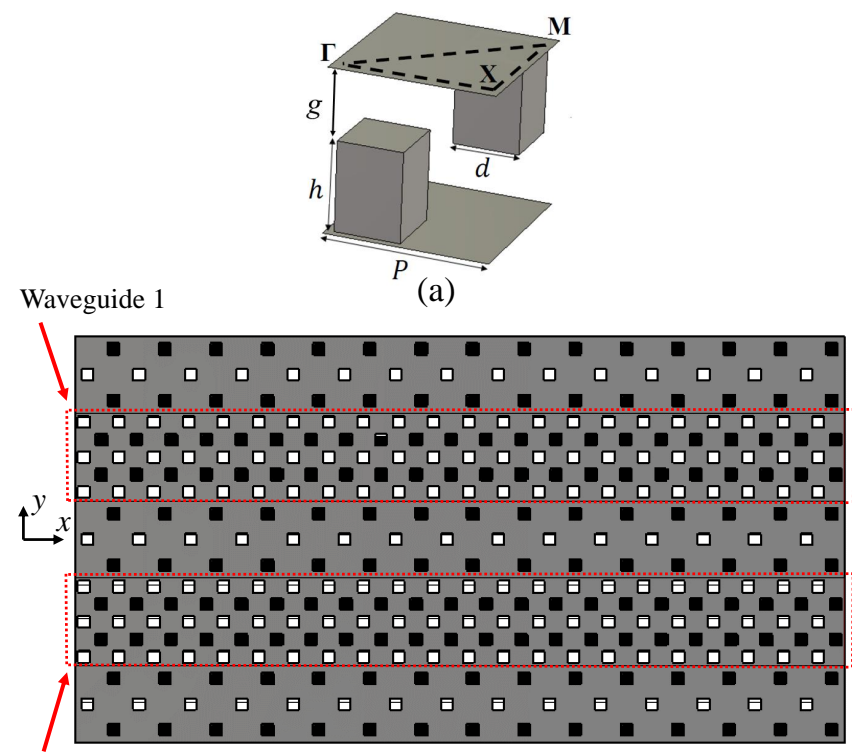

Waveguide 2

(b)

Fig. 1. (a) Unit cell of a glide-symmetric pin loaded parallel plate waveguide. (b) The top view of the structure with two waveguides that is cut in half along the $x y$ plane.

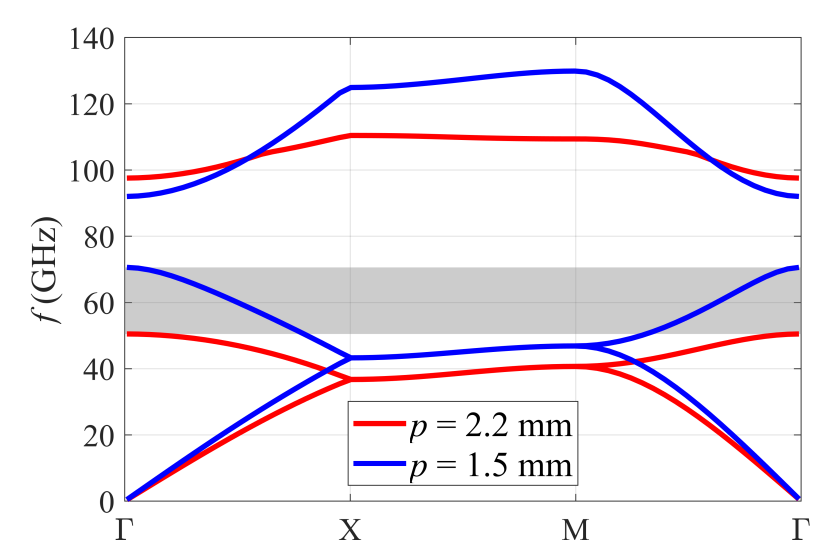

Fig. 2. Dispersion diagram of the unit cell in Fig. 1 (a) with parameters $h=1.2 \mathrm{~mm}, d=0.5 \mathrm{~mm}$ and $g=0.25 \mathrm{~mm}$ for two different values of period $p=1.5 \mathrm{~mm}$ and $p=2.2 \mathrm{~mm}$.

frequency range. In particular, we have moved the starting point of the stopband to a lower frequency. The frequency region formed between the current and the former starting frequencies shows the potential operating frequency range of the waveguide. This region is shown in Fig. 2 with a gray rectangle. Fig. 2 demonstrates a stopband from 51 to $98 \mathrm{GHz}$ for the EBG while the waveguide region has a passband from 0 to $65 \mathrm{GHz}$. As a result, in the frequency range of 51 to 65 $\mathrm{GHz}$ (gray region in the figure), the wave can propagate in the waveguide regions while being completely shielded at the sides by the EBGs.

We now discuss how the gap $g$ between the pins and the opposite plate can be used to design a switch with which we can enable or prevent the propagation along the line. As suggested earlier, this can be achieved by mechanically moving the plates of the PPW closer to or further away from each other. We plot in Fig. 3 the dispersion diagram of our unit cell with a gap of $g=0.1 \mathrm{~mm}$ (red color) and compare it to the diagram of $g=0.25 \mathrm{~mm}$ (blue color). Fig. 3 (a) demonstrates that decreasing the gap has in fact increased the stopband region of the waveguide while keeping the center frequency of the stopband approximately constant at $80 \mathrm{GHz}$. 54 to $65 \mathrm{GHz}$ is in the stopband of the red curve $(g=0.1 \mathrm{~mm})$ while it is in the passband of the blue curve $(g=0.25 \mathrm{~mm})$. This suggests that by mechanically changing the gap from $g=0.25 \mathrm{~mm}$ to $g=0.1 \mathrm{~mm}$, the wave propagation once enabled in our structure in the frequency range of 54 to $65 \mathrm{GHz}$ will now be blocked. In Fig. 3 (b) we plot the dispersion diagram of the EBG with $g=0.1 \mathrm{~mm}$ (red color) and compare it to when $g=0.25 \mathrm{~mm}$ (blue color). The result demonstrates that the the frequency range of 54 to $65 \mathrm{GHz}$ still belongs to the stopband of the EBG. In conclusion, by changing the gap $g$ between the two values $g=0.1 \mathrm{~mm}$ and $g=0.25 \mathrm{~mm}$, the EBG always stays a bandgap medium in the desired frequency range while the waveguide region alternates between a bandgap and a passband medium.

In Fig. 4 we visualize how the electromagnetic power flow propagates or attenuates according to the gap value $g$. Fig. 4 (a) displays the power flow of the wave in the structure of Fig. 1 (b) when the gap is $g=0.25 \mathrm{~mm}$ and the top waveguide is excited from the left side. This confirms that the electromagnetic fields are confined in the waveguide region while propagating from the left port to the right port. It also confirms that 3 rows of pins in the EBG are enough to suppress the propagation of the wave in the cross-sectional direction. Therefore, no power leaks into the adjacent waveguide. This qualitatively shows that it is possible to achieve good isolation between different waveguides in a multiparallel switching structure for instance. In contrast, Fig. 4 (b) shows the power flow in the same structure when $g=0.1 \mathrm{~mm}$ and the top waveguide is excited again from the left side. One can see that there is no propagation of the electromagnetic wave in this case as the wave is attenuated by the periodic structure. The structure is then completely mismatched.

\section{B. Phase Shifter}

We have already noticed in Figs. 3 (a) and 3 (b) how changing the gap $g$ affects the stopband and passband frequencies. A closer look at these two figures shows that due to the shift of the stopband, the phase constant of the waveguides with two different gap values are different. Therefore, it is possible to support waves with different phase constants in the two waveguides of Fig. 2 (b) if we choose a different gap $g$ for each waveguide. This allows for design of a phase shifter. Again, the fact that we can mechanically adjust the gap $g$ suggests the feasibility of a reconfigurable design. To show this idea, Fig. 5 (a) displays the vertical component of the electrical field at a time instant at $59 \mathrm{GHz}$ when the gap equals $g=0.2 \mathrm{~mm}$ 


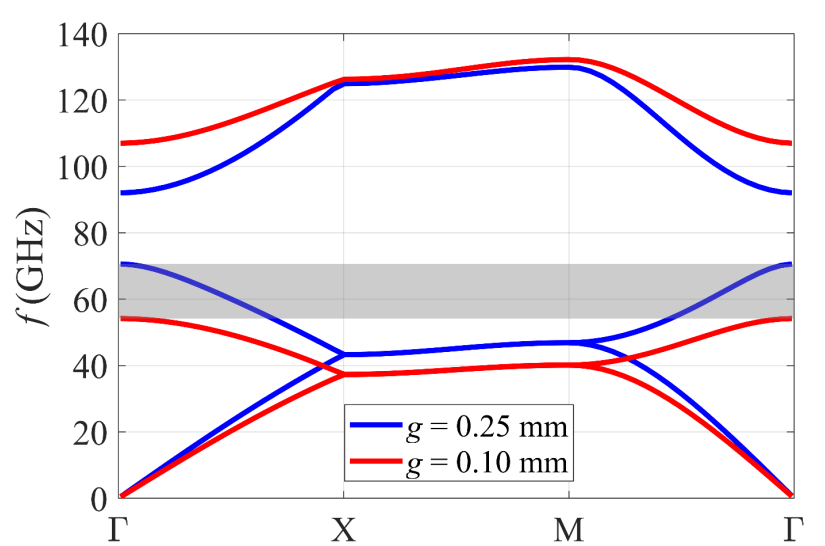

(a)

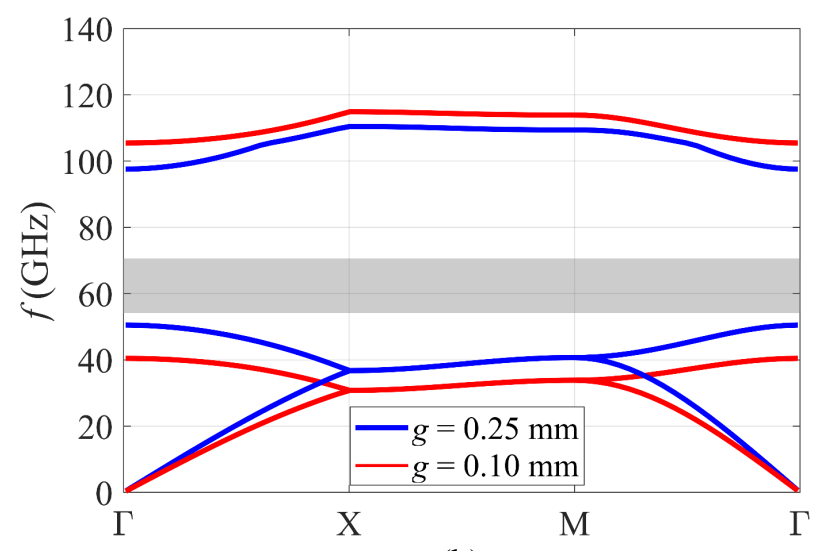

(b)

Fig. 3. Dispersion diagram of the unit cell in Fig. 1 (a) with parameters $h=1.2 \mathrm{~mm}, d=0.5 \mathrm{~mm}$ and (a) $p=1.5 \mathrm{~mm}$ (in the waveguide). (b) $p=$ $2.2 \mathrm{~mm}$ (in the EBG).

while Fig 5 (b) plots the same values when the gap equals $g=0.17 \mathrm{~mm}$. A comparison of the zeros of the fields between the two figures clearly shows that the propagating wave in Fig. 5 (a) has a smaller wavenumber than the wave in Fig. 5 (b). This confirms how two different guides with different gaps can be used to create a desired phase shift between the waves.

\section{CONCLUSION}

The mechanical reconfiguration of a glide-symmetric waveguide has been shown to be an effective way to achieve a two-state on-off structure. This can be used for the design of a low-loss RF switch. Indeed, the signal in this topology does not propagate through semi-conductor material but only in air. The only losses are the ohmic losses of the metallic structure. These glide-symmetric waveguides could be therefore useful to switch between multiple input port of mm-wave multibeam antennas. Furthermore, the capability to achieve mechanically reconfigurable phase shifters with the proposed geometry has also been addressed.

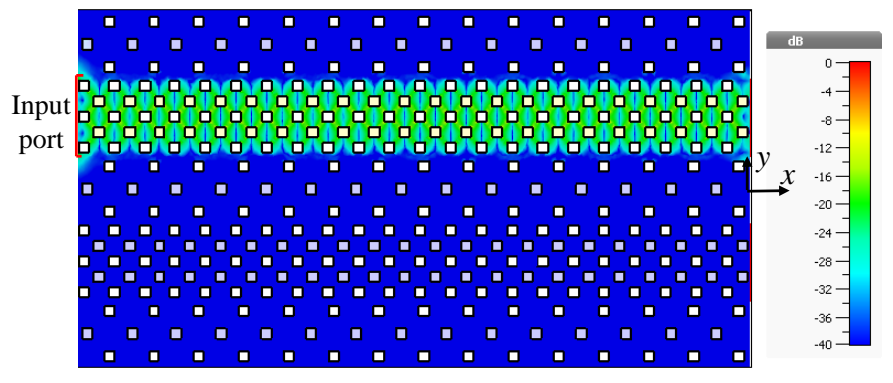

(a)

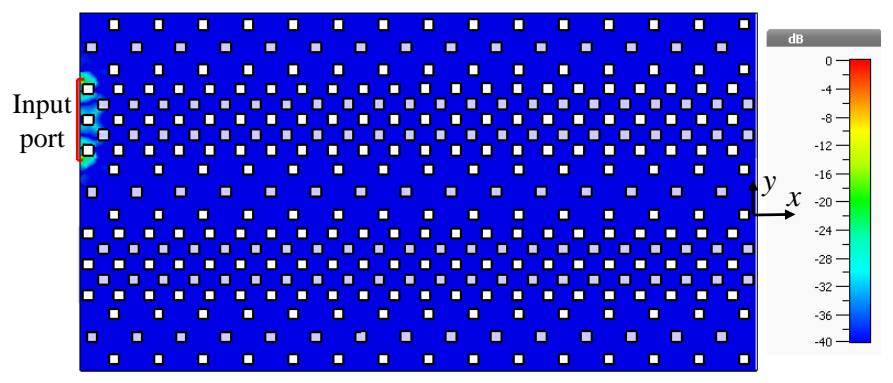

(b)

Fig. 4. Power flow in the structure in Fig. 1 (b) when the top waveguide is excited from the left side: (a) $g=0.25 \mathrm{~mm}$. (b) $g=0.1 \mathrm{~mm}$.

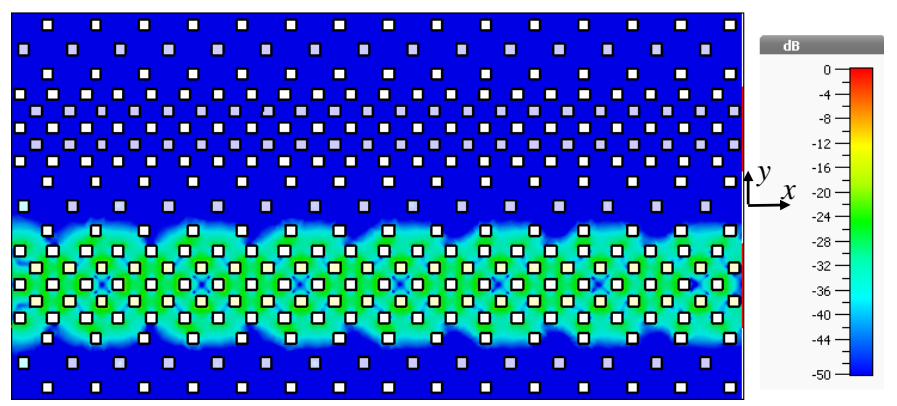

(a)

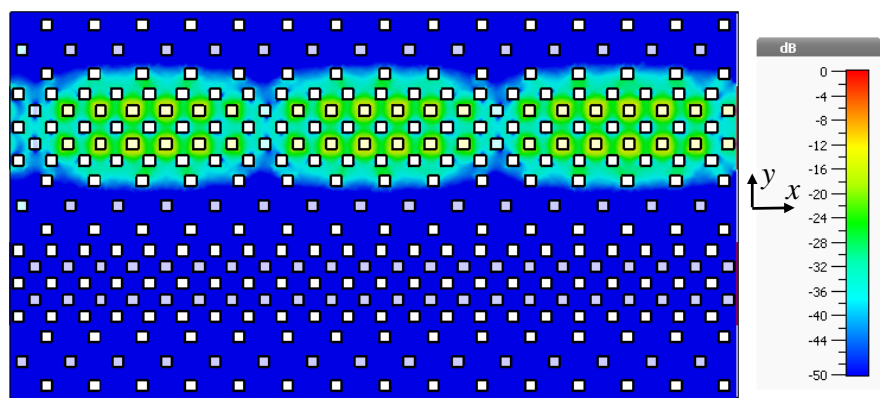

(b)

Fig. 5. Vertical component of the electrical field in the structure of Fig. 1 (b): (a) The bottom waveguide is excited with $g=0.20 \mathrm{~mm}$. (b) The top waveguide is excited with $g=0.17 \mathrm{~mm}$. 


\section{ACKNOWLEDGMENT}

This work was supported by the French governement under the ANR grant HOLeYMETA ANR JCJC 2016 ANR-16CE24-0030 and by Sorbonne Universités under the EMERGENCE 2016 grant MetaSym. This work was performed within the NOVIS60 project supported by the CEFPRA (IndoFrench Center for the Promotion of Advanced Research).

\section{REFERENCES}

[1] M. G. Silveirinha, C. A. Fernandes, and J. R. Costa, "Electromagnetic characterization of textured surfaces formed by metallic pins," IEEE Transactions on Antennas and Propagation, vol. 56, no. 2, pp. 405415,2008

[2] S. Maci, G. Minatti, M. Casaletti, and M. Bosiljevac, "Metasurfing: Addressing waves on impenetrable metasurfaces," IEEE Antennas and Wireless Propagation Letters, vol. 10, pp. 1499-1502, 2011.

[3] E. Rajo-Iglesias and P.-S. Kildal, "Numerical studies of bandwidth of parallel-plate cut-off realised by a bed of nails, corrugations and mushroom-type electromagnetic bandgap for use in gap waveguides," IET microwaves, antennas \& propagation, vol. 5, no. 3, pp. 282-289, 2011.

[4] P.-S. Kildal, E. Alfonso, A. Valero-Nogueira, and E. Rajo-Iglesias, "Local metamaterial-based waveguides in gaps between parallel metal plates," IEEE Antennas and wireless propagation letters, vol. 8, pp. 8487, 2008.

[5] P.-S. Kildal, A. U. Zaman, E. Rajo-Iglesias, E. Alfonso, and A. ValeroNogueira, "Design and experimental verification of ridge gap waveguide in bed of nails for parallel-plate mode suppression," IET Microwaves, Antennas \& Propagation, vol. 5, no. 3, pp. 262-270, 2011.

[6] A. Vosoogh and P.-S. Kildal, "Corporate-fed planar 60-ghz slot array made of three unconnected metal layers using amc pin surface for the gap waveguide," IEEE Antennas and Wireless Propagation Letters, vol. 15, pp. 1935-1938, 2015.

[7] M. Bagheriasl, O. Quevedo-Teruel, and G. Valerio, "Bloch analysis of artificial lines and surfaces exhibiting glide symmetry," IEEE Transactions on Microwave Theory and Techniques, vol. 67, no. 7, pp. 2618-2628, 2019.

[8] K. Liu, F. Ghasemifard, and O. Quevedo-Teruel, "Broadband metasurface luneburg lens antenna based on glide-symmetric bed of nails," in 2017 11th European Conference on Antennas and Propagation (EUCAP), March 2017, pp. 358-360.

[9] O. Quevedo-Teruel, M. Ebrahimpouri, and M. N. M. Kehn, "Ultrawideband metasurface lenses based on off-shifted opposite layers," IEEE Antennas and Wireless Propagation Letters, vol. 15, pp. 484-487, 2015

[10] M. Ebrahimpouri, O. Quevedo-Teruel, and E. Rajo-Iglesias, "Design guidelines for gap waveguide technology based on glide-symmetric holey structures," IEEE microwave and wireless components letters, vol. 27, no. 6, pp. 542-544, 2017.

[11] M. Ebrahimpouri, E. Rajo-Iglesias, Z. Sipus, and O. Quevedo-Teruel, "Cost-effective gap waveguide technology based on glide-symmetric holey ebg structures," IEEE Transactions on Microwave Theory and Techniques, vol. 66, no. 2, pp. 927-934, Feb 2018.

[12] E. Rajo-Iglesias, M. Ebrahimpouri, and O. Quevedo-Teruel, "Wideband phase shifter in groove gap waveguide technology implemented with glide-symmetric holey ebg," IEEE Microwave and Wireless Components Letters, vol. 28, no. 6, pp. 476-478, June 2018. 